DOI: 10.34185/1991-7848.itmm.2020.01.045

\title{
PARALLEL IMPLEMENTATION OF THE COMBINED ALGORITHM OF THE BRANCH AND BOUND METHOD
}

\author{
Ivaschenko Valeriy, Shvachych Gennady, \\ Konovalenkov Vladimir, Khristyan Vladimir \\ National Metallurgical Academy of Ukraine, Dnipro, Ukraine
}

\begin{abstract}
Parallel implementation of a combined branch-and-bound algorithm for the knapsack problem are considered. An approach combining parallel implementations of the branch-and-bound method and the heuristic search is proposed and implemented. Basic attention is focused on the questions of research of efficiency and acceleration for calculations due to the increase of the cluster system knots. As a result of the proposed approach, a organization scheme of the combined algorithm of distributed computing was obtained. The approach proposed in this paper saves the developers' efforts by reapplying common parts of the algorithm to solve various problems of optimization. In fact, one can implement a common solution scheme for different platforms once, and later use only problem-dependent modules for a specific class of problems.
\end{abstract}

Keywords: PERSONAL PERSONALIZED CALCULABLE CLUSTER, PARALLEL COMPUTING, BRANCH-AND-BOUND METHOD, KNAPSACK PROBLEM, LOCAL OPTIMIZATION.

Introduction. In economic and mathematical modeling there often occurs a situation when the economic system under study has too complicated structure. Indeed, the researcher is often faced with its description multidi.mensionality. Such problems include, for instance, problems of market segmentation, forecasting of market conditions, studying and forecasting of economic depression, analysis and forecasting of social and economic phenomena, and so fourth. Besides, we note that multidimensional methods are indispensable for researchers in both marketing and management.

This class of problems is extremely important for development of the economy in general, in this regard, the development of effective methods of solving these problems and their implementation through packages of applications is a pressing scientific and practical problem. At the same time, a distinctive feature of solving these types of problems is their high computational complexity. However, in our opinion, effective research of such systems can be carried out only on the basis of 
application of multiprocessor computer systems [1]. Nowadays, significant interest has grown in the construction of multiprocessor parallel computing systems, based on standard publicly available technologies and components.

Research main material statement. This paper aims to develop methods of distributed modeling in economics, proposed by the authors in a number of papers. The solution to the global optimization problem is illustrated by the backpack problem solution example by branch and border method. From the economics point of view, another interpretation of the backpack problem is more relevant, when orders (or production variants of lots of certain goods) are considered as items, and as utility - the profit from the particular order execution, and as weight - the cost of the order. In general, the backpack problem is a mathematical model of many applied problems and not only of economics, but the theory of management, cryptography, etc. In this regard, the development of effective and reliable methods for solving such problems is extremely important.

There are two main families of methods for solving this problem: exact and heuristic. Accurate methods guarantee the optimality of the solution found. This class includes various variants of the branch and bound method (BBM), clipping method, etc. Accurate methods are characterized by high complexity that quite often does not allow them to be used when solving real problems. Heuristic methods are based on assumptions about the optimal solution properties [2]. Unlike accurate heuristic methods, do not guarantee the optimality of the solution found. However, with limited computing resources, heuristics are often the only way to find a solution. Hybrid methods are also used, when heuristic methods are used to find solutions and accurate methods to prove optimality. The effectiveness of hybrid methods bespeak that heuristic algorithms often have a higher convergence rate to the optimum than the exact methods.

In this paper, hybrid methods based on BBM and heuristic algorithm got further development. The BBM essence is to decompose many admissible solutions on a subset (subproblems) with eliminating subproblems that are not deliberately promising. In order to accelerate the computations, a parallel algorithm of the method based on BBM was developed. The idea of parallel implementation of BBM is to distribute the subproblems generated in the decomposition process by respective processors of the cluster computing system. The vertices of the branch tree are sent directly between the processors of the computing system. In this case, at each step of the BBM, the problem under study is decomposed into two. Further a binary 
subproblem tree is formed, where the arcs are directed from the initial problem to the subproblems that get formed during branching. This creates a decomposition tree. Non-promising end vertices of a tree are excluded by screening rules. The promising end vertex is branching and is replaced by those subproblems that arose from the decomposition. The modeling process is over provided all the finite vertices are sifted away, i.e. no further branching is possible.

The computation is accelerated by the heuristic algorithm application. The heuristic approach allows significantly reducing the computation time, and the solution found will be close to the optimal one or coincide with it altogether. The proposed implementation of computational process allows efficiently implementing the screening procedure for unpromising solutions and thus accelerates the solution of the global optimization problem.

Conclusions. As a result of the proposed approach, a organization scheme of the combined algorithm of distributed computing was obtained. The approach proposed in this paper saves the developers' efforts by reapplying common parts of the algorithm to solve various problems of optimization. In fact, one can implement a common solution scheme for different platforms once, and later use only problemdependent modules for a specific class of problems.

A series of computational experiments was conducted; which aimed at investigating the efficiency of the developed parallel combined algorithm. For comparison, a variant of the backpack problem was considered with implementation of the Horwitz-Sahni method. Several computational experiments have been conducted to demonstrate the benefits of the proposed approach that combines the optimal "acceleration-efficiency" option for a multiprocessor computing system.

\section{References}

1. V.P. Ivaschenko Improving the efficiency of the multiprocessor system through inline interface network aggregation / V.P. Ivaschenko, G.G. Shvachych, E.V. Ivaschenko, V.V. Busygin // System technologies. N 2 (115) - Dnipro, 2018.- P.84-92.

2. Ivaschenko V.P. Effective algorithms for solving the transient coefficient of high accuracy order / V.P. Ivaschenko, G.G. Shvachych, E.V. Ivaschenko, V.V. Busygin // System technologies. N 4 (117) - Dnipro, 2018.- P.86 - 94.4. 